\title{
AOR
}

Selected Papers of \#AolR2020:

The 22nd Annual Conference of the

Association of Internet Researchers

Virtual Event / 13-16 Oct 2021

\section{COVID-19 ACROSS PLATFORM SOCIETIES: EXPLORING THE PANDEMIC THROUGH WEIBO AND TWITTER}

\author{
Zheng Yang \\ Department of Sociological Studies; The University of Sheffield \\ Stefania Vicari \\ Department of Sociological Studies; The University of Sheffield
}

\section{Information, and COVID-19}

Platform studies often offer a Western-centric theorization of the roles and values of contemporary social media platforms. The 'digital universalism' (Chan, 2014) underlying this work is however being increasingly called into question by research directly interested in the 'regionality of platforms' (Steinberg and Li, 2017). In fact, this research is specifically drawing attention to the way platforms - with their uses, cultures and values - are shaped by regional settings. The Chinese digital ecosystem has so far offered the most fertile ground for the growth of non-western centric platform studies. For instance, Wang and Lobato have called for a 'spatialized platform theory' - one both paying attention to platforms' historical and contextual trajectories and assessing how these trajectories influence platforms within frames of reference that may not align with the liberal democratic model of free speech and free markets (2019: 367).

The contemporary call for relational and contextual approaches to digital platforms is also extremely central to our understanding of participatory dynamics across contemporary 'platform societies'. Several studies have investigated the emergence of critical public opinion within the networked publics developing on social media platforms. For instance, research has shown that Twitter's socio-technical infrastructure has allowed the coming to prominence of actors traditionally defined as 'non-elite', namely ordinary users (Hermida, 2015; Jackson \& Foucault Welles, 2015, 2016; Meraz \& Papacharissi, 2013). These changes have not entirely suppressed traditional power structures; they have turned traditional media systems into 'hybrid' (Chadwick, 2017) ecosystems. 
The 'public sphere' constructed by Chinese digital media has however been shown to hold completely different characteristics from those found in the Western society. In their early Internet research, Kalathil and Boas (2001) highlighted the impact of authoritarian regimes on Internet use, drawing attention to how, in those contexts, digital media have often been used to both extend authoritarian reach and push forward national development. Contemporary Chinese social media have been shown to play a peculiar role in relation to participatory practices (Bomsel, 2014) - with their political function undergoing dynamic development (Yang \& Calhoun, 2007). This role is shaped by local dynamics that influence user practices related to, for instance, how opinions are expressed, what content is being produced, distributed and used and which users become influential in gatekeeping dynamics, namely in selecting what content becomes most visible (Yang, 2016). Ultimately, existing research shows that the Chinese platform ecosystem has developed through the tightening of China's state control over digital media and at the intersection of corporate and public interests.

In line with research pointing at the need to regionalise platform studies approaches and challenge digital universalism, in this paper we explore digital participatory cultures across platform societies. We do so by presenting a comparative exploration of participatory dynamics developing on Weibo and Twitter when the Covid-19 pandemic started, respectively, in China and in the West. We explore these dynamics by specifically focusing on the sentiment, the topics and the (human) gatekeepers characterising content posted over a 48-hour period. We used Python and the Twitter Capture and Analysis Tool (TCAT) to collect data respectively from Weibo and Twitter. We then applied sentiment analysis, semantic network analysis and repost network mapping to a random selection of 301,000 weibo posts and tweets.

Our findings show that at the outbreak of the Covid-19 pandemic in China and in the West, Weibo and Twitter played very different roles as participatory platforms. Authoritarian propaganda mechanisms clearly emerged on Weibo, with the accounts of major official media like People's Daily engaging in 'thought work' (Kalathil \& Boas, 2001). Not only did state propaganda nudge behaviour change through key national media outlets; it also developed propaganda networks through individual user accounts. In particular, slogans with nationalist tendencies, such as "million people united as one man" (万众一心) and "our wills unite like a fortress" (众志成城) were forwarded, absorbed, re-created and disseminated by individual 'Internet celebrities'. It is these 'propaganda' mechanisms that most likely also led to polarizing sentiment in Weibo conversations about the pandemic. These dynamics did not apply to the 'democratized' Western world, where other forms of influence were exerted, as signalled by the centrality of former US President Donald Trump's account in gatekeeping practices. In fact, our findings show that elite accounts that can affect the political agenda played a central role in influencing the conversation and, most likely, the overall framing of the events - not necessarily in line with public health policies or scientific evidence.

In conclusion, at the start of the pandemic, Weibo primarily displayed a number of 'topdown' processes while Twitter incorporated a range of dynamics: elite influence, horizontal flows, conflicting opinions and political framings of the events. In China, platforms can act as 'propaganda tools' used to unite and promote public policies under 
the control of the government and the guidance of state-controlled mainstream media. In Western societies, platforms seem to work more as 'messy public squares' that allow the expression of different personal opinions, though under the influence of legacy media and public figures with different political agendas. In fact, democratic governments often find themselves struggling to impose effective regulation on platforms that have grown rapidly without their immediate oversight (Van Dijck et al., 2018). Ultimately, our findings point to the need to further develop platform studies that take into account the local contexts in which platforms are used, developed and regulated (Willems, 2020).

\section{Reference}

Bomsel, O. (2014). Is China a Weibo democracy. International Relations and Diplomacy, 2(2), 120-132.

Chadwick, A. (2017). The hybrid media system: Politics and power. Oxford University Press.

Chan, A. (2013). Networking peripheries: Technological futures and the myth of digital universalism. MIT Press.

Hermida, A. (2015). Power plays on social media. Social Media +Society, 1. doi:10.1177/2056305115580340

Jackson, S., \& Foucault Welles, B. (2015). Hijacking \#myNYPD: Social media dissent and networked counterpublics. Journal of Communication, 65, 932-952.

Jackson, S., \& Foucault Welles, B. (2016). \#Ferguson is everywhere: Initiators in emerging counterpublic networks. Information, Communication \& Society, 19, 397-418.

Kalathil, S., \& Boas, T. C. (2001). The Internet and state control in authoritarian regimes: China, Cuba and the counterrevolution. First Monday, 6(8). https://doi.org/10.5210/fm.v6i8.876

Meraz, S., \& Papacharissi, Z. (2013). Networked gatekeeping and networked framing on \#Egypt. The International Journal of Press/Politics, 18, 138-166.

Steinberg, M., \& Li, J. (2017). Introduction: Regional platforms. Asiascape: Digital Asia, 4(3), 173-183.

Van Dijck, J., Poell, T., \& De Waal, M. (2018). The platform society: Public values in a connective world. Oxford University Press.

Wang, W.Y. and Lobato, R., (2019). Chinese video streaming services in the context of global platform studies. Chinese Journal of Communication, 12(3), pp.356-371. 
Willems, W. (2020). Beyond platform-centrism and digital universalism: the relational affordances of mobile social media publics. Information, Communication \& Society, 1-17.

Yang, G. (2016). The Red Guard generation and political activism in China. Columbia University Press.

Yang, G., \& Calhoun, C. (2007). Media, civil society, and the rise of a green public sphere in China. China Information, 21(2), 211-236. 